\title{
Decaimentos nucleares em uma câmara de nuvens
}

\author{
(Nuclear decays in a cloud chamber)
}

\author{
Caio Laganá⿴囗十 \\ Instituto de Física, Universidade de São Paulo, São Paulo, SP, Brasil \\ Recebido em 9/11/2012; Aceito em 2/3/2013; Publicado em 26/9/2013
}

\begin{abstract}
Imagens de decaimentos- $\alpha$ de uma fonte radioativa de Amerício-241, obtidas com uma câmara de nuvens de baixo custo, são utilizadas como motivação para o desenvolvimento de conceitos ligados à passagem de partículas carregadas pela matéria e na discussão de um modelo simples de estrutura nuclear. O espectro energético do ${ }^{241} \mathrm{Am}$ é analisado a partir de imagens obtidas com o experimento.
\end{abstract}

Palavras-chave: câmara de nuvens, decaimentos nucleares, interação da radiação com a matéria.

Images from $\alpha$-decays of a radioactive 241-Americium source, obtained with a low cost cloud chamber, are used as motivation for the development of concepts related to passage of charged particles through matter and in the discussion of a simple model for nuclear structure. The ${ }^{241} \mathrm{Am}$ spectrum is analyzed using images obtained with the experiment.

Keywords: cloud chamber, nuclear decays, energy loss.

\section{Introdução}

Modelos de estrutura nuclear são um rico campo para aplicações de conceitos de mecânica quântica em um curso de graduação em física. O mais elementar dos casos talvez seja o uso do conceito de tunelamento na construção de modelos para decaimentos nucleares. Além de fundamental do ponto de vista teórico, o estudo de decaimentos nucleares pode ser facilmente vinculado a uma prática experimental, onde partículas decaídas podem ser observadas utilizando-se uma câmara de nuvens.

Este trabalho apresenta imagens de decaimentos nucleares de uma fonte de Amerício-241 observados com uma câmara de nuvens de baixo custo, utilizando-as como motivação para discussão de um modelo simples de estrutura nuclear. Além disso, alguns conceitos ligados a passagem de partículas carregadas pela matéria são desenvolvidos, com ênfase na sua aplicação em física experimental de altas energias e na física médica. O espectro energético das partículas- $\alpha$ oriundas da fonte de Amerício é analisado a partir de imagens obtidas com o experimento.

\section{A câmara de nuvens}

A câmara de nuvens é um detector onde traços produzidos por partículas subatômicas podem ser vistos a olho nu. O esquema de sua montagem é apresentado

\footnotetext{
${ }^{1}$ E-mail: caiolagana@gmail.com.

Copyright by the Sociedade Brasileira de Física. Printed in Brazil.
}

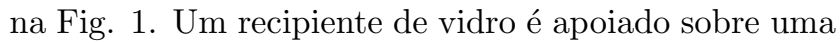
mesa de cobre, parcialmente submersa em uma mistura de álcool e gelo seco. Um pedaço de papel embebido com álcool, afixado na parte superior interna do vidro, faz com que o recipiente fique constantemente preenchido com vapor de álcool. Na região mais próxima à placa de cobre, até uma altura de $\sim 1 \mathrm{~cm}$, o vapor de álcool encontra-se supersaturado, devido à baixa temperatura do gelo seco. Quando uma partícula carregada atravessa o vapor supersaturado de álcool, ela ioniza as moléculas que estão em seu caminho, induzindo a formação de gotículas, que por sua vez dão origem a um traço que, quando iluminado por LED's, pode ser observado a olho nu.

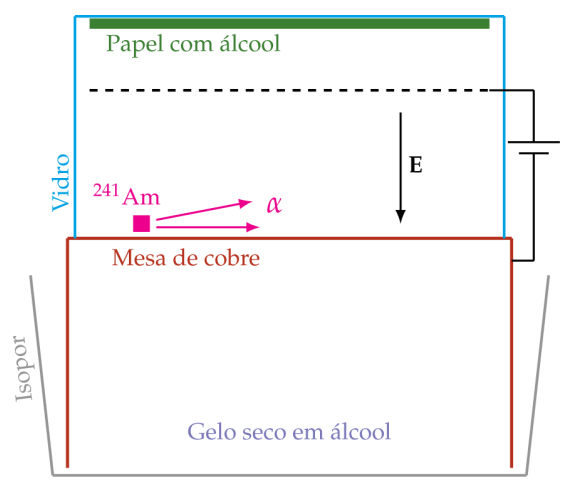

Figura 1 - Esquema de montagem da câmara de nuvens. As Refs. [Ш] e [ర] apresentam maiores detalhes acerca da construção e funcionamento do arranjo. 
Um circuito amplificador extraído de uma "raquetemata-mosquito" fornece alta voltagem entre uma grade de metal e a mesa de cobre. O campo elétrico assim produzido remove íons positivos do vapor, conferindo melhor resolução aos traços formados na câmara.

As principais fontes de partículas que podem ser observadas em uma câmara de nuvens são raios cósmicos e materiais radioativos. Este trabalho utilizou uma fonte de Amerício-241 retirada de um detector de fumaça como fonte de partículas- $\alpha$. Em um trabalho anterior [四], uma câmara de nuvens muito similiar à descrita acima foi utilizada para investigação de raios cósmicos.

A grande atividade de radiação- $\alpha$ do ${ }^{241} \mathrm{Am}$ pode comprometer a visualização dos traços formados na câmara de nuvens. Para contornar este problema, a fonte foi blindada com papel alumínio, e um furo de diâmetro $\sim 1 \mathrm{~mm}$, feito com uma agulha, fez com que um feixe relativamente escasso de partículas fosse produzido. A intensidade deste feixe mostrou-se adequada para observação de traços na câmara de nuvens.

\section{Partículas- $\alpha$ observadas na câmara de nuvens}

Traços deixados na câmara de nuvens por partículas- $\alpha$ oriundas de uma fonte de ${ }^{241} \mathrm{Am}$ são apresentados na Fig. ఇ]. No canto superior esquerdo desta figura, é mostrado o traço de um elétron de baixa energia $(\sim 50 \mathrm{keV})$ provavelmente ejetado de um átomo por um múon produzido por um raio cósmico [ד].

Considerando que o processo de formação de traços é o mesmo para elétrons e partículas- $\alpha$ - criação de gotículas a partir da ionização do meio - uma simples questão pode ser colocada com base na Fig. 『: por que o traço deixado por partículas- $\alpha$ é tão mais espesso que aquele formado por elétrons? A proposição de tal pergunta em uma sala de aula pode levar a uma rica discussão a respeito do conceito de taxa de deposição de energia, efeito utilizado na identificação de partículas em experimentos de altas energias e no tratamento de tumores em física médica.

Ao atravessar determinado material, seja ele sólido, líquido ou gasoso, partículas carregadas perdem energia predominantemente através da ionização do meio. Na câmara de nuvens, esta ionização é responsável pela formação dos traços das partículas, e é tanto maior quanto maior a carga e massa da partícula incidente.

A Fig. \$1 mostra um gráfico da taxa de deposição de energia $(d E / d x)$ de elétrons, prótons e partículas- $\alpha$ em vapor de água, como função de sua energia cinética (escolheu-se vapor de água pois estes eram os dados disponíveis em [3] mais semelhantes às condições presentes na câmara de nuvens). Com base neste gráfico, é possível observar que partículas alfas depositam quase três ordens de magnetude mais energia que elétrons, fato que dá origem ao seu traço espesso característico.

Em experimentos de altas energias, a coleta dos íons gerados por partículas carregadas ao atravessarem determinado material é um mecanismo amplamente utilizado na sua identificação. A "espessura" do traço é traduzida na amplitude de um sinal elétrico, proporcional a $d E / d x$. Sabendo-se o momento da partícula (a partir de sua curvatura em um campo magnético), sua massa pode ser determinada através da fórmula de Bethe [四], que, em um regime moderadamente relativístico $(E \gtrsim m)$, é geralmente parametrizada por

$$
\frac{d E}{d x}=K \frac{m^{2}}{\mathbf{p}^{2}}+C
$$

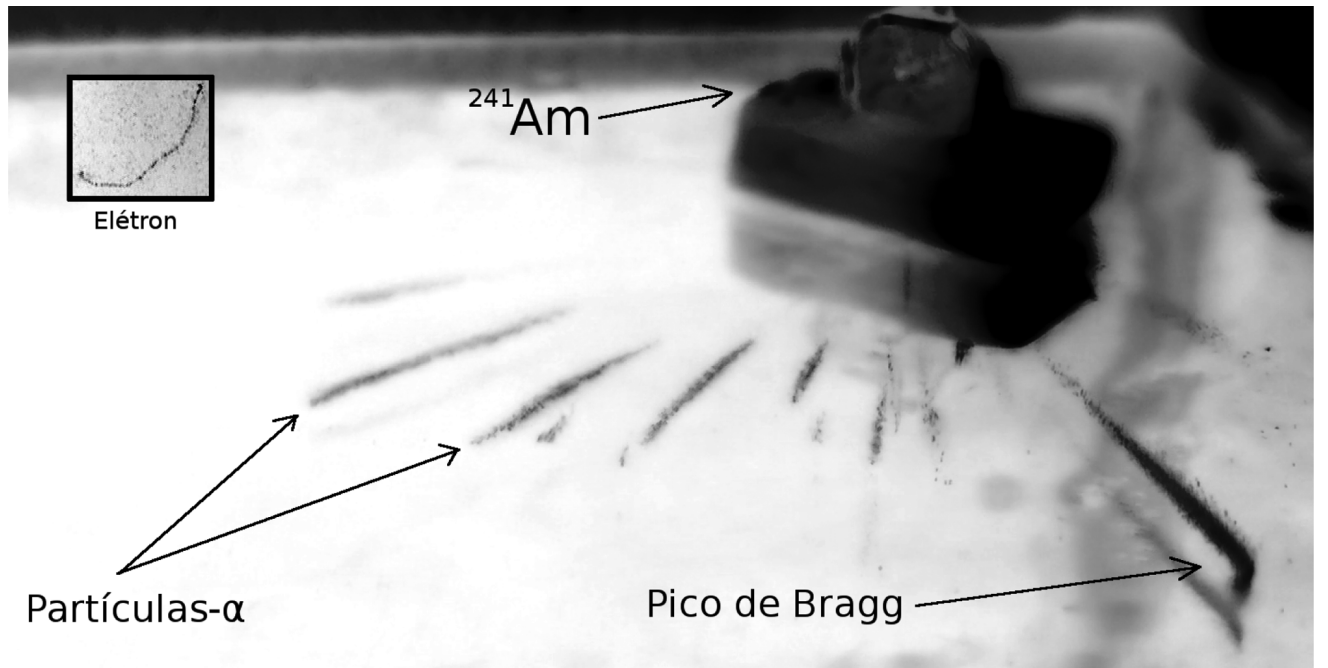

Figura 2 - Partículas- $\alpha$ oriundas de uma fonte de ${ }^{241} \mathrm{Am}$ observadas em uma câmara de nuvens. No canto superior esquerdo, o traço deixado por um elétron. 


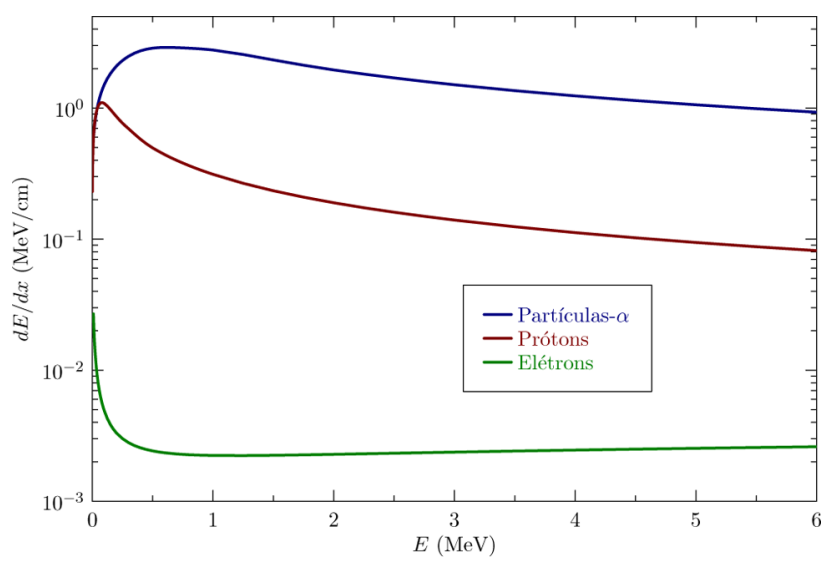

Figura 3 - Taxa de deposição de energia $(d E / d x)$ de elétrons, prótons e partículas- $\alpha$ em vapor de água, como função de sua energia cinética. O gráfico foi feito com dados extraídos da Ref. [3].

Em regimes não relativísticos $(E \ll m)$, como é o caso das pariculas- $\alpha$ da fonte de ${ }^{241} \mathrm{Am}$ neste trabalho, diversas correções devem ser levadas em conta na fórmula de Bethe. Tais correções incorporam efeitos de escala atômica na interação da partícula incidente com o meio, fornecendo uma expressão teórica para $d E / d x$ válida até $E \sim 2 \mathrm{MeV}$ para partículas- $\alpha$, e até $\sim 0.5 \mathrm{MeV}$ para prótons [3]. Abaixo dessas energias, a perda de energia é geralmente obtida a partir de ajustes empíricos a dados experimentais. De modo geral, portanto, a forma mais imediata de entender a deposição de energia na escala de poucos $\mathrm{MeV}$ é através da leitura de gráficos experimentais como os da Fig. [1.

Além da espessura do traço, a taxa de deposição de energia determina também a distância máxima que uma partícula de massa $m$ e energia $E$ percorre antes de parar: $D_{\max }$, também chamada de alcançe (range). Um gráfico do alcançe de prótons e partículas- $\alpha$ em vapor de água como função de sua energia cinética pode ser visto na Fig. 四.

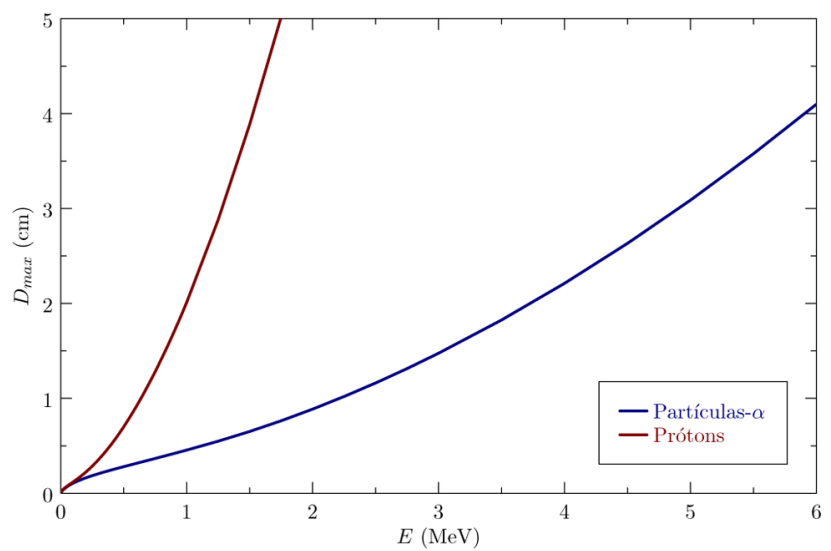

Figura 4 - Alcançe de prótons e partículas- $\alpha$ em vapor de água como função de sua energia cinética.
A energia depositada por uma partícula de alcançe $D_{\max }$ varia ao longo de sua trajetória - de modo geral, quanto menor a velocidade da partícula, mais energia ela depositará, pois o tempo de interação com o meio aumenta. Espera-se, portanto, que a maior fração de energia depositada por uma partícula seja na porção final de sua trajetória, pouco antes de ela parar completamente.

A Fig. 1 mostra o perfil de $d E / d x$ ao longo da trajetória de partículas- $\alpha$ de $5 \mathrm{MeV}$ de energia cinética, e prótons de $1.5 \mathrm{MeV}$. O pico que surge ao final das trajetórias é conhecido como pico de Bragg. Descoberto em 1903, o pico é hoje em dia utilizado na física médica, onde a calibração precisa da energia inicial de um feixe de partículas faz com que a maior parte da energia depositada por elas ao incidir o corpo humano seja, por exemplo, sobre de uma região tumorosa, matando assim as células cancerígenas.

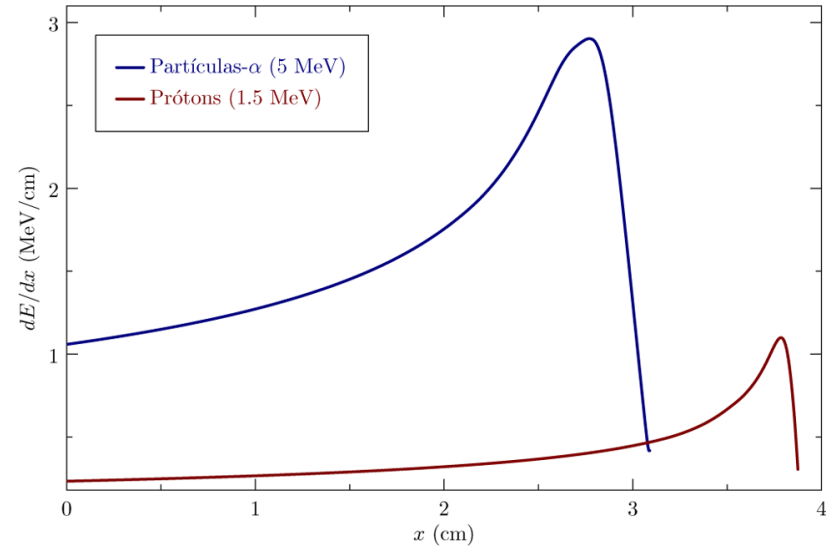

Figura 5 - Taxa de deposição de energia ao longo da trajetória de partículas- $\alpha$ de $5 \mathrm{MeV}$ de energia cinética e prótons de $1.5 \mathrm{MeV}$. As curvas foram obtidas a partir de integração numérica dos gráficos da Fig. $\mathbf{B}$

Um olhar atento aos traços da Fig. 『 revela a presença inequívoca do pico de Bragg em uma situação real: a espessura do traço da direita aumenta ao longo de sua trajetória, terminando com um máximo. Este comportamento traduz, de forma clara e direta, a curva apresentada na Fig. 6. Vale dizer que este é o único traço da fotografia em que o pico de Bragg pode ser visto, pois ele está mais próximo aos LED's que iluminam a câmara, localizados no canto direito do experimento.

Independentemente do perfil de $d E / d x$ ao longo da trajetória, é possível determinar a energia com que uma partícula- $\alpha$ foi emitida da fonte de Amerício a partir do tamanho de seu traço, utilizando-se apenas do gráfico da Fig. 田. Este fato motiva uma interessante atividade que pode ser proposta em sala de aula: a investigação do espectro energético do Amerício. 


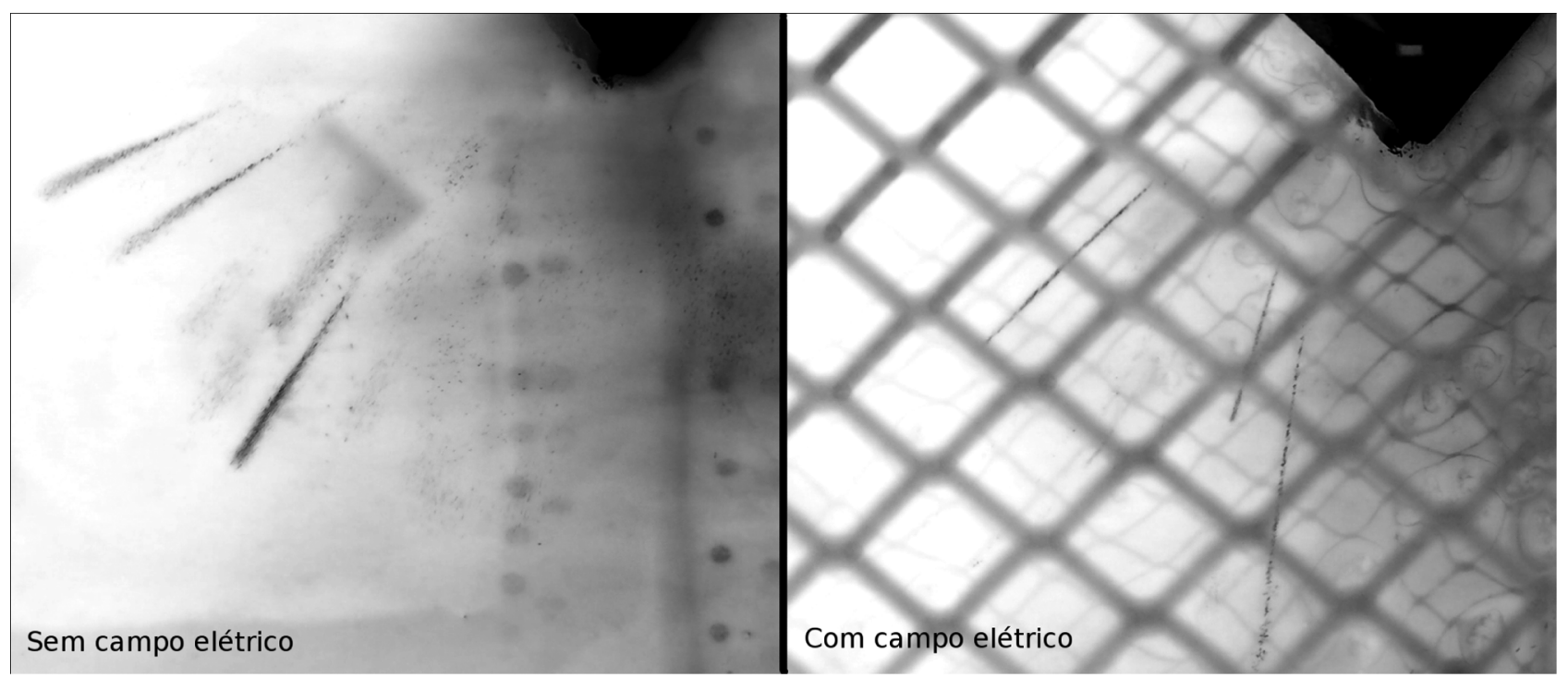

Figura 6 - Partículas- $\alpha$ observadas a um ângulo de $90^{\circ}$ com a base da câmara. Do lado direito, é possível ver a grade onde é aplicada a alta tensão, e o efeito que o campo elétrico causa na qualidade dos traços.

A Fig. $\square$ mostra um histograma do alcançe de partículas- $\alpha$ emitidas da fonte de ${ }^{241} \mathrm{Am}$. O histograma foi construido a partir da análise de filmagens da câmara de nuvens, feitas a um ângulo de $90^{\circ}$ com a placa de cobre. Uma imagem típica observada dessa forma é mostrada na Fig. [ (lado direito). A figura também ilustra o efeito que o campo elétrico provoca sobre a qualidade dos traços. É interessante notar que a extrapolação dos traços até o ponto onde eles se cruzariam fornece uma coordenada relativamente precisa para a localização da fonte de ${ }^{241} \mathrm{Am}$. Tal fato pode ser utilizado para descartar eventuais traços (de origem cósmica) que atravessem a câmera durante a análise das filmagens.

Para construção do histograma da Fig. [, a aproximadamente cada 5 quadros de filme (tempo para que um novo conjunto de traços decaídos surja), o tamanho dos traços das partículas eram medidos com o software ImageJ. Uma régua de calibração foi inserida para converter $D_{\max }$ de pixels para centímetros. O histograma corresponde a aproximadamente 3 minutos de filmagem.

A partir do histograma de Fig. [, pode-se estimar o alcançe médio de parítulas- $\alpha$ vindas do ${ }^{241} \mathrm{Am}$ como $\left\langle D_{\max }\right\rangle=3.21 \pm 0.48 \mathrm{~cm}$. De acordo com o gráfico da Fig. $⿴$, este alcançe corresponde a uma energia cinética de $5.13 \pm 0.45 \mathrm{MeV}$. Muito embora este valor seja compatível com o atualmente aceito, de $5.486 \mathrm{MeV}$ [G], ele está $6.5 \%$ abaixo do esperado. Como explicar tal discrepância?

No arranjo da câmara de nuvens utilizada neste trabalho, ao menos três fatores limitam a precisão das medidas:

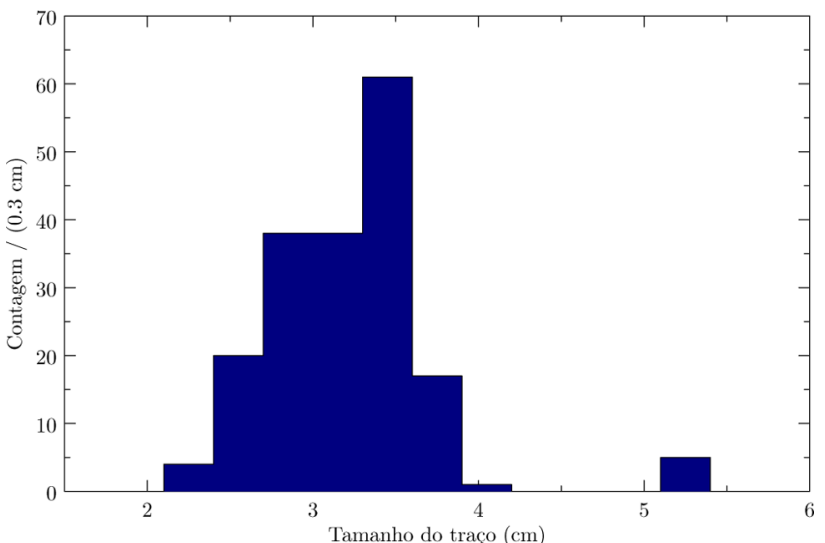

Figura 7 - Histograma da distância máxima percorrida por partículas- $\alpha$ emitidas da fonte de ${ }^{241} \mathrm{Am}$, obtido a partir da análise de filmagens da câmara de nuvens.

(i) A região de detecção sensível a formação de traços, onde se encontra o vapor supersaturado de álcool, corresponde, aproximadamente, a um paralelepípedo de dimensões $10 \mathrm{~cm} \times 10 \mathrm{~cm} \times 1 \mathrm{~cm}$. Como as filmagens são feitas a partir da parte superior da câmara (contra o plano- $x y$ ), os desvios verticais (ao longo do eixo- $z$ ) sofridos pelas partículas são ignorados. Tal fato pode subestimar o tamanho real de um traço em até $5 \%$.

(ii) O processo de ionização do vapor de álcool é um fenômeno estatístico (a física subjascente está associada, em última análise, a probabilidades de transição do tipo $\mid\left.\left\langle\alpha+\right.$ átomo $\left|H_{\text {int }}\right| \alpha+$ íon $\rangle\right|^{2}$, determinadas pela mecânica quântica). O caráter estatístico da ionização de uma trajetória (chamado de straggling) leva a uma flutuação da ordem de $2 \%$ no alcançe das partículas [ [ $[$ ]. 
(iii) A fonte de Amerício utilizada neste trabalho possui uma certa espessura. Dessa forma, uma partícula- $\alpha$ que tenha sido emitida a uma dada profundidade da superfície pode perder energia no próprio material. Este problema ocorre pois a fonte foi retirada de um detector de fumaça, onde o cuidado com a espessura não é levado em conta. Fontes de calibração precisas são muito finas, de forma a minimizar este problema.

Os fatores citados acima introduzem incertezas nas medidas do alcançe de partículas- $\alpha$, e são responsáveis pela grande largura do histograma. Além disso, os itens (i) e (iii) fazem com que o alcançe medido seja ligeiramente menor do que seu valor real, o que explica o desvio de $\left(E_{\text {real }}-E_{\text {medido }}\right) / E_{\text {real }}=6.5 \%$.

Como mostrado nos parágrafos acima, o processo de análise das filmagens da câmara de nuvens partilha de diversas técnicas de análise presentes em física experimental de altas e baixas energias. Deve-se levar em conta, por exemplo, erros sistemáticos introduzidos devido às particularidades do arranjo; a eficiência na detecção das partículas; a definição de um critério de seleção de dados que seja imparcial (minimum bias). A análise de filmagens de uma câmara de nuvens em sala de aula pode ser, portanto, um excelente laboratório para discussão de conceitos de física experimental entre alunos de graduação.

Todos os aspectos relacionados à instrumentação de detecção e interação de partículas carregadas com a matéria discutidos até então são de suma importância para o entendimento dos decaimentos nucleares observados na câmara de nuvens. Tais aspectos representam, a meu ver, um alicerce sobre o qual o desenvolvimento de conceitos abstratos acerca da natureza do fenômeno físico pode ser realizado. O corpo de tais conceitos abstratos é o que usualmente leva o nome de "física teórica", enquanto seu alicerce pode ser entendido como a "física experimental". Muito embora a separação entre estas duas áreas seja útil para o desenvolvimento e especialização de técnicas particulares a cada uma delas, acredito que uma visão conjunta destes dois ramos do conhecimento seja essencial para bom entendimento de qualquer fenômeno físico. À luz desta ideia, a próxima seção é motivada pela seguinte pergunta: como explicar os decaimentos nucleares da fonte de Amerício observados na câmara de nuvens?

\section{Modelo de decaimento nuclear}

Modelos de Estrutura Nuclear lidam com "sistemas de partículas cujo número não é nem pequeno o suficiente que possibilite soluções diretas, nem grande o suficiente que permita o uso de métodos estatísticos" [6]. Por conta disso, soluções analíticas exatas que partem de primeiros princípios são, para todos os efeitos, até o momento, inexistentes. Ainda assim, alguns modelos sim- plificados são capazes de descrever certos fenômenos nucleares observados experimentalmente, fornecendo insights a respeito de dinâmicas do mundo subatômico. O poço de potencial quadrado é um desses modelos.

Sugerido pela primeira vez por Gamow em 1928 [], o modelo do poço quadrado considera que o potencial efetivo sentido por uma partícula- $\alpha$ no interior de um núcleo pesado é semelhante àquele representado pela Fig. \: uma região fortemente atrativa e de curto alcance $\left(r<r_{1} \sim 7 \mathrm{fm}\right)$, fruto de interações nucleares e responsável pela ligação dos nucleons, seguida de uma repulsão Coulombiana entre a carga $2 e$ da partícula- $\alpha$ e a carga Ze no núcleo pesado. É interessante notar que a repulsão Coulombiana está presente também no interior do núcleo, mas é desprezível em vista da magnetude das forças nucleares a distâncias tão pequenas.

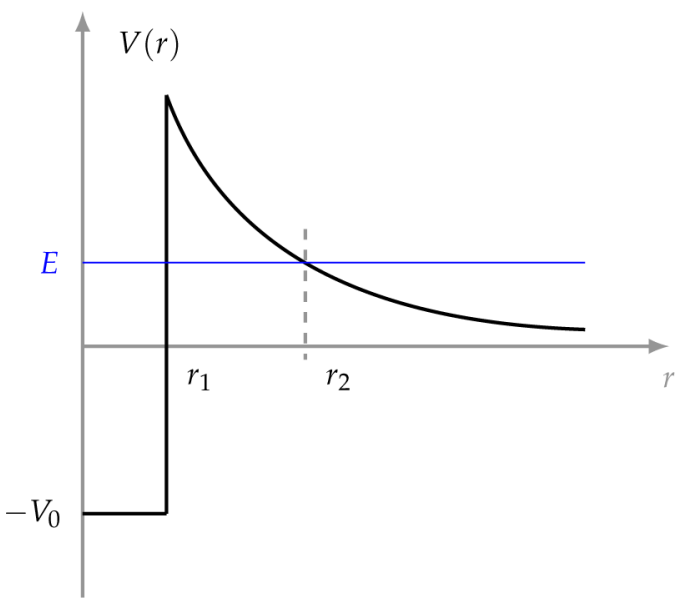

Figura 8 - Potencial efetivo sentido por uma partícula- $\alpha$ na presença de um núcleo pesado.

Se a partícula- $\alpha$ possui energia $E$ tal que $0<E<$ $V\left(r_{1}\right)$, existe uma probabilidade de ela tunelar através das paredes do potencial, e propagar-se livremente para fora do núcleo. Este processo de tunelamento é responsável pelo decaimento de partículas- $\alpha$ em núcleos pesados, e pode ser quantificado a partir da resolução da equação de Schroedinger para a partícula- $\alpha$ sujeita ao potencial da Fig. $\mathbf{8}$. O desenvolvimento completo do problema pode ser visto na Ref. [ళ]. Como bibliografia introdutória, recomendo o Cap. 8 da Ref. [G]. Os principais resultados serão resumidos a seguir.

A vida-média de uma partícula sujeita a um potencial do tipo "barreira" (onde uma região classicamente não acessível impede sua propagação livre) é dada por

$$
\tau=\frac{2 r_{1}}{v} e^{2 \sigma}
$$

O fator $\sigma$ contém a informação acerca da particular forma de $V(r)$

$$
\sigma=\int_{r_{1}}^{r_{2}} \sqrt{2 m\left(2 Z \frac{\alpha \hbar c}{r}-E\right)} d r
$$


enquanto que o termo $2 r_{1} / v$ representa a frequência com que a partícula incide sobre a parede do potencial em $r=r_{1}$, sendo $v$ sua velocidade.

Nota-se, a partir das Eqs. (Ш) e (四), que a vidamédia de determinado elemento depende da energia com que a partícula- $\alpha$ é emitida. A resolução da integral permite afirmar [0] que esta dependência é do tipo

$$
\ln \tau=a \frac{Z}{\sqrt{E}}-b \sqrt{Z r_{1}}
$$

Para fixar ideias, imaginemos diferentes isótopos do Urânio $(Z=92)$, onde a única dependência de $\ln \tau$ é, para todos os efeitos, a energia da partícula emitida. Tal energia varia de isótopo para isótopo (como a interação entre os nucleons é sempre atrativa, espera-se que isótopos com menor número de neutrons tenham partículas- $\alpha$ menos ligadas, portanto mais energéticas).

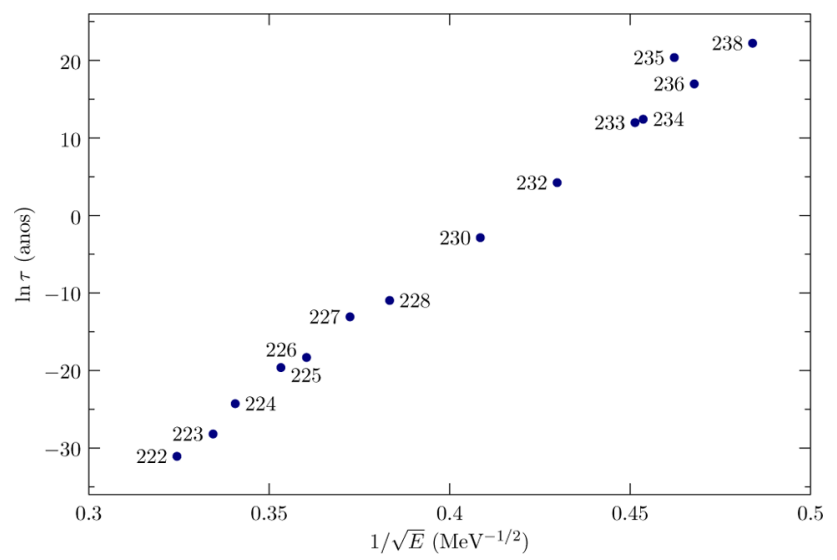

Figura 9 - Logaritmo da vida média de isótopos do Urânio em função do inverso da raíz quadrada da energia cinética da partícula- $\alpha$ decaída. Dados extraídos da Ref. [س]]

Um gráfico do logarítmo da vida média de diversos isótopos do Urânio $(A=222 \cdots 238)$, como função do inverso da raíz quadrada da energia da partícula- $\alpha$ emitida, pode ser visto na Fig. Q. É possível observar que, de modo geral, a dependência de $\ln \tau \operatorname{com} 1 / \sqrt{E}$ segue o comportamento linear previsto pela Eq. (田).

A capacidade de unificar 50 ordens de magnetude no valor de vidas-médias em uma única expressão é um forte indício de que o entendimento de decaimentos nucleares a partir do mecanismo de tunelamento está correto.

\section{Conclusão}

Este trabalho utilizou imagens de decaimentos- $\alpha$ de uma fonte de Amerício-241, obtidas com uma câmara de nuvens de baixo custo, como motivação para o desenvolvimento de conceitos ligados a passagem de partículas carregadas pela matéria e na discussão de um modelo simples de estrutura nuclear.

Uma noção básica foi dada sobre como a taxa de deposição de energia é utilizada na identificação de partículas em experimentos de altas energias, e como este mesmo mecanismo dá origem ao pico de Bragg, efeito utilizado na física médica. A partir de um histograma do alcançe de partículas- $\alpha$, construído através da análise de imagens obtidas com a câmara de nuvens, foi possível investigar o espectro energético do ${ }^{241} \mathrm{Am}$. A análise dos dados mostrou-se uma potencial atividade a ser realizada entre alunos de graduação para discussão de conceitos presentes em física experimental de altas e baixas energias.

\section{Agradecimentos}

Agradeço ao prof. Helio Takai pelas preciosas sugestões dadas ao longo da construção do experimento, bem como na análise e interpretação dos resultados.

\section{Referências}

[1] C. Laganá, Revista Brasileira de Ensino de Física 33, 3 (2011).

[2] C. Laganá, sites.google.com/site/caiolagana/ cloud-chamber-construcad.

[3] National Institute of Standards and Technology, physics.nist.gov/PhysRefData.

[4] K. Nakamura and Particle Data Group, Journal of Physics G 37, 075021 (2010).

[5] J.K. Boggild, Nature 161, 810 (1948).

[6] Greiner Maruhn, Nuclear Models (Ed. Springer, New York, 1995).

[7] G. Gamow, Z. Phys. 51, 204 (1928).

[8] Barry R. Holstein, Am. J. Phys. 64, 1061 (1996).

[9] David J. Griffiths, Introduction to Quantum Mechanics (Pearson Prentice Hall, Upper Saddle River, 2005).

[10] National Nuclear Data Center, www.nndc.bnl.gov/ chart. 